\title{
Detection of viral RNA fragments in human iPSC cardiomyocytes following treatment with extracellular vesicles from SARS-CoV-2 coding sequence overexpressing lung epithelial cells
}

Youjeong Kwon ${ }^{1 \dagger}$, Sarath Babu Nukala ${ }^{1 \dagger}$, Shubhi Srivastava ${ }^{1 \dagger}$, Hiroe Miyamoto ${ }^{1}$, Nur Izzah Ismail ${ }^{2,3,4}$, Jordan Jousma', Jalees Rehman ${ }^{1,5}$, Sang-Bing Ong ${ }^{2,3,4^{*}}$, Won Hee Lee ${ }^{6^{*}}$ and Sang-Ging Ong ${ }^{1,5^{*}}$ (i)

\begin{abstract}
Coronavirus disease 2019 (COVID-19) caused by the severe acute respiratory syndrome coronavirus 2 (SARS-CoV-2) is a global pandemic. The prevalence/severity of COVID-19 is higher among patients with cardiovascular risk factors. Despite the expression of angiotensin-converting enzyme 2 (ACE2), a receptor for SARS-CoV-2 infection, in cardiomyocytes, there has been no conclusive evidence of direct viral infection although the presence of viral genome within COVID-19 patients' hearts has been reported. Here, we overexpressed SARS-CoV-2 genes in A549 lung epithelial cells. We then isolated extracellular vesicles (EVs) and detected the presence of viral RNA within these EVs. We observed that human induced pluripotent stem cell-derived cardiomyocytes (hiPSC-CMs) are receptive to these EVs, and viral genes were detectable in the cardiomyocytes. Accordingly, the uptake of viral RNAharboring EVs led to an upregulation of inflammation-related genes in hiPSC-CMs. Thus, our findings indicate that SARS-CoV-2 RNA containing EVs represents an indirect route of viral RNA entry into cardiomyocytes.
\end{abstract}

Keywords: COVID-19, Extracellular vesicles, iPSCs, Stem cells, Cardiomyocytes

\footnotetext{
*Correspondence: sangbingong@cuhk.edu.hk; whlee@email.arizona.edu; sangging@uic.edu

${ }^{\dagger}$ Youjeong Kwon, Sarath Babu Nukala and Shubhi Srivastava contributed equally to this work.

${ }^{2}$ Centre for Cardiovascular Genomics and Medicine, Lui Che Woo Institute of Innovative Medicine, Chinese University of Hong Kong (CUHK), Hong Kong, Hong Kong SAR

${ }^{6}$ Department of Basic Medical Sciences, University of Arizona College of

Medicine - Phoenix, 425 N 5th St., Bldg ABC1 Rm 426, Phoenix, AZ 85004, USA

'Department of Pharmacology, The University of Illinois College of Medicine, 909 S Wolcott Ave, COMRB 4097, Chicago, IL 60612, USA

Full list of author information is available at the end of the article
}

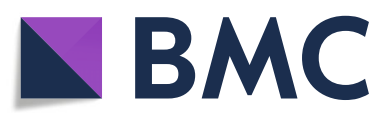

(- The Author(s). 2020 Open Access This article is licensed under a Creative Commons Attribution 4.0 International License, which permits use, sharing, adaptation, distribution and reproduction in any medium or format, as long as you give appropriate credit to the original author(s) and the source, provide a link to the Creative Commons licence, and indicate if changes were made. The images or other third party material in this article are included in the article's Creative Commons licence, unless indicated otherwise in a credit line to the material. If material is not included in the article's Creative Commons licence and your intended use is not permitted by statutory regulation or exceeds the permitted use, you will need to obtain permission directly from the copyright holder. To view a copy of this licence, visit http://creativecommons.org/licenses/by/4.0/ The Creative Commons Public Domain Dedication waiver (http://creativecommons.org/publicdomain/zero/1.0/) applies to the data made available in this article, unless otherwise stated in a credit line to the data. 
Dear Editor,

Since the initial outbreak in China, coronavirus disease 2019 (COVID-19) which is caused by severe acute respiratory syndrome coronavirus 2 (SARS-CoV-2) has evolved into a global pandemic. While COVID-19 affects both healthy individuals and those with comorbid conditions such as cardiovascular diseases, the severity and risk of adverse outcomes of COVID-19 are especially pronounced in the latter [1]. Furthermore, patients with COVID-19 have also been reported to exhibit increased levels of cardiac biomarkers, suggestive of cardiac injury [2]. However, it remains unclear whether exacerbated cardiac injury seen in COVID-19 patients results directly from viral SARS-CoV-2 infection of the myocardium or indirectly from the complications of COVID-19. Cardiomyocytes express angiotensin-converting enzyme 2 (ACE2), the SARS-CoV-2 binding site [3]. However, there is no evidence of direct viral infection of cardiomyocytes to date clinically, although the presence of myocardial inflammation and viral particles among the interstitial cells of the myocardium has been reported [4] and viral RNA has also been detected in some COVID-19 patients' hearts [5]. The majority of cells in the body are known to release lipid bilayer membrane vesicles, also known as extracellular vesicles (EVs), that are capable of transferring various genetic materials including viral RNAs to other recipient cells $[6,7]$. Therefore, in the present work, we hypothesized that SARS-CoV-2-infected cells such as airway epithelial cells secrete EVs carrying viral genetic material that may be taken up by cardiomyocytes and establish an indirect route of SARS-CoV-2 genetic material transmission.

To test whether the viral RNA of SARS-CoV-2 can be transmitted via EVs into cardiomyocytes without the need for direct infection, we transduced A549 lung epithelial cells with lentivirus encoding selected SARSCoV-2 proteins [8] (Fig. 1a). A549 cells were chosen as a model cell type since COVID-19 appears to mainly infect respiratory tract cells in patients. SARS-CoV-2 genes encoding for two non-structural proteins (Nsp1 and $N s p 12$ ) and two structural proteins (envelope $E$ and nucleocapsid $N$ ) were used for this proof-of-principle study. We opted not to include the spike $(S)$ protein, which is required for receptor binding and viral entry, in order to focus on EV-mediated transfer of viral fragments into recipient cardiomyocytes that are independent of S-protein-mediated direct viral entry. The use of lentivirus overexpressing viral subunits also allowed us to distinguish EV-mediated SARS-CoV-2 RNA transfer from canonical virus infection since $\mathrm{EV}$ preparations inevitably contain infectious virions due to the overlap in size.

Quantitative RT-PCR on total RNA extracted from A549 cells $48 \mathrm{~h}$ after lentivirus transduction confirmed the successful overexpression of viral RNAs encoding for Nsp1, Nsp12, E, and N compared to a control empty vector (Fig. 1b). To isolate EVs released by A549 cells, the supernatant of A549 cells grown in a culture medium supplemented with exosome-depleted FBS for $48 \mathrm{~h}$ was collected for EV purification. Immunoblotting of EV preparations confirmed the enrichment of the EV markers CD63, CD81, and TSG101 although we did note the presence of albumin most likely due to the PEG-based isolation method used (Fig. 1c). Additional NanoSight analysis of EVs from control, Nsp1, and Nsp12 overexpressing A549 cells confirmed the size of EVs ranged from 50 to $300 \mathrm{~nm}$ with no significant difference in terms of particle concentration between all measured groups although there was an increasing trend in the Nsp12 overexpressing A549 cells group (Figure S1).

We next asked whether the RNAs encoding for SARSCoV-2 are packaged into purified EVs of A549 cells. qRT-PCR revealed the presence of mRNA in purified EVs for each of the four tested SARS-CoV-2 genes (Fig. 1d). We performed a separate validation for Nsp1 and Nsp12 in EVs isolated using a different method based on immuno-magnetic CD63 beads and successfully confirmed the presence of both tested genes in the isolated EVs (Figure S2). To further substantiate the presence of viral genes within EVs, we treated our Nsp1 EV preparation with RNase/protease. As expected, treatment with RNase alone or protease + RNase led to minimal loss of Nsp1, while the addition of detergent led to significant degradation of the enclosed Nsp1 (Figure S3). To study if human cardiomyocytes are able to uptake EVs, we labeled EVs with a fluorescent dye ExoGlow and incubated them $(100 \mu \mathrm{g}$ based on protein quantification) with human induced pluripotent stem cells-derived cardiomyocytes (hiPSC-CMs, $1 \times 10^{6}$ cells). Following 6 $\mathrm{h}$ of $37^{\circ} \mathrm{C}$ incubation and washout of unbound EVs, we observed the presence of labeled EVs in treated hiPSCCMs, which was not observed when the cells were incubated with the negative control (PBS without EVs stained with ExoGlow), confirming the successful binding/uptake of EVs by the recipient hiPSC-CMs (Fig. 1e and Figure S4). Moreover, after exposure of hiPSC-CMs to A549 EVs for $24 \mathrm{~h}$, we detected all four tested viral RNAs in the hiPSC-CMs, but we could not detect any significant levels of viral RNAs in hiPSC-CMs treated with control EVs (Fig. 1f). In a separate set of experiments, we exposed hiPSC-CMs to conditioned media collected from Nsp1-overexpressing A549 cells $\left(1 \times 10^{7}\right.$ cells) with and without concurrent treatment of GW4869 $(5 \mu \mathrm{M})$, an inhibitor of exosome generation. The expression of Nsp1 in hiPSC-CMs was significantly blunted when GW4869 was present, consistent with the involvement of EVs in RNA transfer (Figure S5). Next, we tested whether the phenomenon of uptake of viral 
A

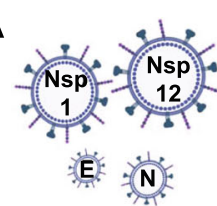

Purified extracellular
vesicles (EVs)

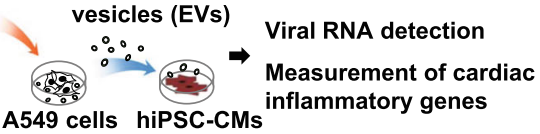

Lentivirus overexpressing

viral subunits
C

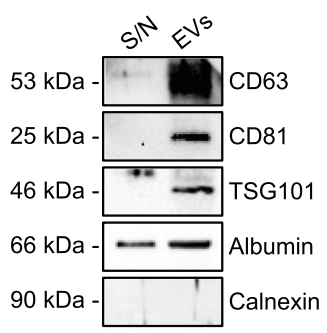

B

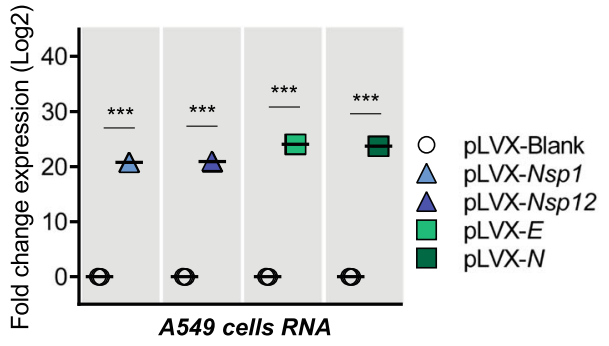

D

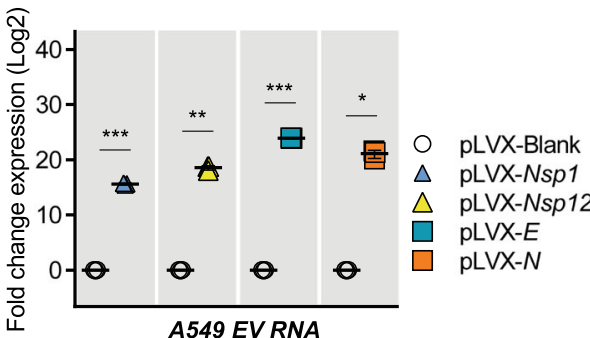

$\mathbf{F}$

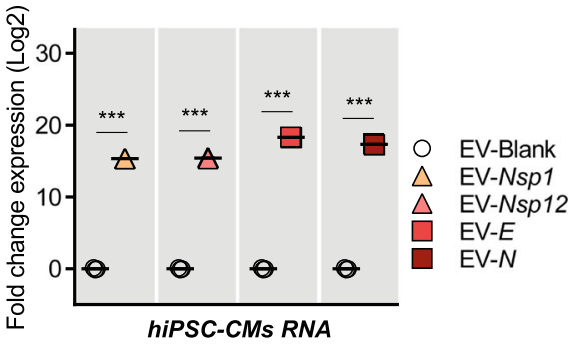

E

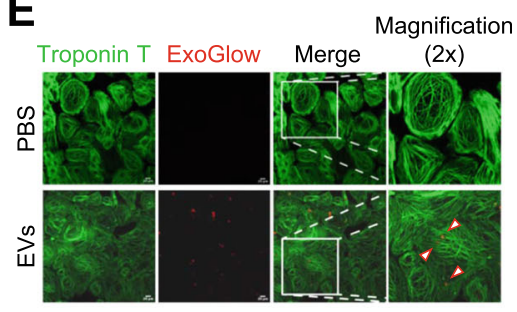

G

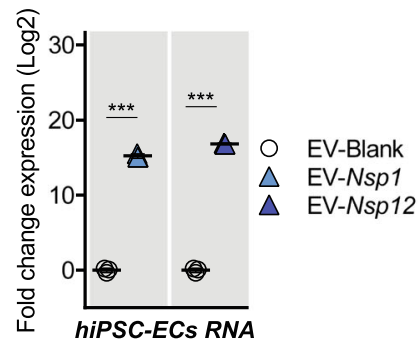

H

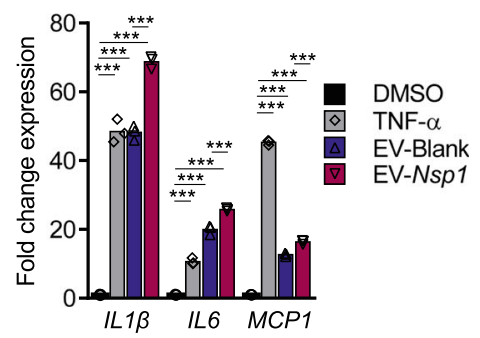

Fig. 1 Detection of SARS-CoV-2 synthetic viral RNA fragments in human induced pluripotent stem cell-derived cardiomyocytes (hiPSC-CMs) and endothelial cells (hiPSC-ECs) treated with EVs. a Schematic depiction of study design. Nsp1, non-structural protein 1; Nsp12, non-structural protein 12; E, envelope protein; N, nucleocapsid protein. b Expression of SARS-Cov-2 genes in A549 lung epithelial cells. A549 cells were infected with indicated lentiviral particles for $48 \mathrm{~h}$ and mRNA levels were measured by qRT-PCR $\left(n=3\right.$, mean \pm S.D). ${ }^{* * *} P<0.001$ versus pLVX-Blank (Student's $t$ test). $\mathbf{c ~ I m m u n o b l o t t i n g ~ o f ~ E V ~ m a r k e r s ~ d e m o n s t r a t i n g ~ e n r i c h m e n t ~ i n ~ t h e ~ E V ~ f r a c t i o n ~ c o m p a r e d ~ t o ~ t h e ~ s u p e r n a t a n t . ~} \mathbf{d}$ SARS-CoV-2 genetic materials (Nsp1, Nsp12, E, and N) were detected in EVs secreted from A549 lung epithelial cells. EVs were purified from A549 cell culture media, and mRNA levels were measured by qRT-PCR $\left(n=3\right.$, mean \pm S.D.). ${ }^{*} P<0.05$; ${ }^{* *} P<0.01$; ${ }^{* * *} P<0.001$ versus pLVX-Blank (Student's $t$ test). e Uptake of ExoGlow-labeled EVs (pseudocolored red) or PBS (negative control) by hiPSC-CMs stained with cardiac troponin T (green) was visualized by confocal imaging. Scale bar $=10 \mu \mathrm{M}$. Small arrows depict detected EVs within cells. $\mathbf{f}$ qRT-PCR was performed to detect the presence of viral genes in hiPSC-CMs following EV uptake. ( $n=3$, mean \pm S.D.). ${ }^{* *} P<0.001$ versus EV-Blank (Student's $t$ test). g qRT-PCR was performed to detect the presence of viral genes Nsp1 and Nsp12 in hiPSC-ECs following EV uptake ( $n=3$, mean \pm S.D.). ${ }^{* *} P<0.001$ versus EV-Blank (Student's $t$ test). h Expression of inflammatory genes in hiPSC-CMs. hiPSC-CMs were treated with EVs released by A549 cells transduced with pLVX-Blank or pLVXNsp1 lentiviral particles for $6 \mathrm{~h}$ and mRNA levels were measured by qRT-PCR $(n=3$, mean \pm S.D.). Tumor necrosis factor-a (TNF- $a, 50 \mathrm{ng} / \mathrm{ml})$ was used as a positive control. ${ }^{* *} P<0.001$ (one-way ANOVA followed by Tukey's multiple comparisons test). $I L 1 \beta$, interleukin $1 \beta$; $I L 6$, interleukin 6 ; $M C P 1$, monocyte chemoattractant protein 1 
gene-containing EVs was also present in hiPSC-derived endothelial cells (hiPSC-ECs) as an additional form of validation. Similar to the hiPSC-CMs, we detected viral RNAs in hiPSC-ECs after A549 EV treatment but not control EVs in a concentration-dependent manner (Fig. 1g, Figure S6). We then assessed whether hiPSCCM exposure to EVs from A549 cells expressing viral RNA increased inflammatory gene expression. It is known that A549 EVs themselves can increase inflammation [9]. We noted that EVs containing Nsp1 further increased the expression of the pro-inflammatory genes $I L 1 \beta$, IL6, and $M C P 1$, suggesting the transferred viral gene may promote inflammation (Fig. $1 \mathrm{~h}$ ).

There are several limitations to our study. Although the precipitation method used in our study for isolating EVs leads to high recovery, it is associated with the recovery of non-EV components such as proteins. However, our additional experiments including immunomagnetic isolation of EVs, RNase/protease treatment, and GW4869 treatment support the enrichment of viral genes within EVs. The ExoGlow dye used cannot fully distinguish between binding and internalization of EVs. It should also be noted that the viral genes used in our study are codon-optimized fragments and expressed at a supraphysiological level. Further experiments are needed to validate if EVs are capable of transferring actual viral RNAs or virions at a physiological level and if the transferred RNAs are biologically active. Lastly, while we have only explored hiPSC-CMs and hiPSC-ECs in this study which both demonstrated uptake of EVs containing viral RNA fragments, we were unable to include a negative control cell type. Overall, our results collectively demonstrated that lung epithelial cells expressing SARS-CoV-2 genes can secrete EVs containing viral RNA fragments that can be detected in cardiomyocytes suggesting an indirect route of viral RNA delivery into cardiac cells via EVs. Transfer of viral RNA via EVs should be considered when studying the widespread multi-organ effects of a SARS-CoV-2 infection that has been reported [10], because it indicates that cells which do not express the SARS-CoV-2 receptor ACE2 might still be vulnerable via the uptake of EVs. Further work is needed to clarify whether the entry of SARS-CoV-2 RNA via EVs is sufficient to induce cell injury and inflammation.

\section{Supplementary Information}

The online version contains supplementary material available at https://doi. org/10.1186/s13287-020-02033-7.

Additional file 1. Supplementary methods, table, and figures.

\section{Abbreviations}

COVID-19: Coronavirus disease 2019; SARS-CoV-2: Severe acute respiratory syndrome coronavirus 2; ACE2: Angiotensin-converting enzyme 2; EVs: Extracellular vesicles; hiPSCs: Human induced pluripotent stem cells;
hiPSC-CMs: Human induced pluripotent stem cell-derived cardiomyocytes; IL1ß: Interleukin 1ß; IL6: Interleukin 6; MCP1: Monocyte chemoattractant protein 1

\section{Acknowledgements \\ We would like to thank Dr. Nevan Krogan for providing the plasmids used in this study.}

\section{Authors' contributions}

Y.K., S.B.O., W.H.L., and S.G.O conceived the project. Y.K., S.B.N., and S.S. performed most of the experiments and data analysis. H.M., N.I.I, and J. J helped with western blotting and virus preparation. S.B.O., W.H.L, and S.G.O supervised the project and wrote the manuscript with support from Y.K., J.R., and other authors. The authors read and approved the final manuscript.

\section{Funding}

J.R is supported by the National Institutes of Health (NIH) R01 HL126516. S.B.O. is supported by a Research Committee's One-off Project Impact Enhancement Fund (PIEF) (Phase 2 - COVID) (PIEF/Ph2/COVID/08) from the Faculty of Medicine, The Chinese University of Hong Kong (CUHK), the Improvement on Competitiveness in Hiring New Faculties Funding Scheme from CUHK, the Centre for Cardiovascular Genomics and Medicine (CCGM), Lui Che Woo Institute of Innovative Medicine CUHK, the Hong Kong Hub of Pediatric Excellence (HK HOPE), Hong Kong Children's Hospital (HKCH), and the Department of Medicine and Therapeutics, Faculty of Medicine, CUHK. W.H.L. is supported by the American Heart Association Scientist Development Grant 16SDG27560003. S.G.O. is supported by the NIH ROO HL130416 and R01 HL148756.

\section{Availability of data and materials}

The datasets used and/or analyzed during the current study are available from the corresponding authors on reasonable request.

Ethics approval and consent to participate

Not applicable

Consent for publication

Not applicable

\section{Competing interests}

The authors declare that they have no competing interests.

\section{Author details}

'Department of Pharmacology, The University of Illinois College of Medicine, 909 S Wolcott Ave, COMRB 4097, Chicago, IL 60612, USA. ${ }^{2}$ Centre for Cardiovascular Genomics and Medicine, Lui Che Woo Institute of Innovative Medicine, Chinese University of Hong Kong (CUHK), Hong Kong, Hong Kong SAR. ${ }^{3}$ Hong Kong Hub of Pediatric Excellence, Hong Kong Children's Hospital, Lab A, 8/F, Tower A, 1 Shing Cheong Road, Kowloon Bay, Kowloon, Kowloon, Hong Kong SAR. ${ }^{4}$ Department of Medicine and Therapeutics, Faculty of Medicine, CUHK, Hong Kong, Hong Kong SAR. ${ }^{5}$ Division of Cardiology, Department of Medicine, The University of Illinois College of Medicine, 909 S Wolcott Ave, COMRB 4097, Chicago, IL 60612, USA.

${ }^{6}$ Department of Basic Medical Sciences, University of Arizona College of Medicine - Phoenix, 425 N 5th St., Bldg ABC1 Rm 426, Phoenix, AZ 85004, USA.

Received: 19 July 2020 Accepted: 17 November 2020

Published online: 30 November 2020

\section{References}

1. Driggin $\mathrm{E}$, et al. Cardiovascular considerations for patients, health care workers, and health systems during the COVID-19 pandemic. J Am Coll Cardiol. 2020;75:2352-71. https://doi.org/10.1016/j.jacc.2020.03.031.

2. Clerkin KJ, et al. COVID-19 and cardiovascular disease. Circulation. 2020;141: 1648-55. https://doi.org/10.1161/CIRCULATIONAHA.120.046941.

3. Crackower MA, et al. Angiotensin-converting enzyme 2 is an essential regulator of heart function. Nature. 2002;417:822-8. https://doi.org/10.1038/nature00786.

4. Tavazzi $G$, et al. Myocardial localization of coronavirus in COVID-19 cardiogenic shock. Eur J Heart Fail. 2020. https://doi.org/10.1002/ejhf.1828. 
5. Wichmann $\mathrm{D}$, et al. Autopsy findings and venous thromboembolism in patients with COVID-19: a prospective cohort study. Ann Intern Med. 2020 https://doi.org/10.7326/M20-2003.

6. Valadi $\mathrm{H}$, et al. Exosome-mediated transfer of mRNAs and microRNAs is a novel mechanism of genetic exchange between cells. Nat Cell Biol. 2007;9: 654-9. https://doi.org/10.1038/ncb1596.

7. Dreux M, et al. Short-range exosomal transfer of viral RNA from infected cells to plasmacytoid dendritic cells triggers innate immunity. Cell Host Microbe. 2012;12:558-70. https://doi.org/10.1016/j.chom.2012.08.010.

8. Gordon DE, et al. A SARS-CoV-2 protein interaction map reveals targets for drug repurposing. Nature. 2020. https://doi.org/10.1038/s41586-020-2286-9.

9. Li X, et al. Lung tumor exosomes induce a pro-inflammatory phenotype in mesenchymal stem cells via NFKB-TLR signaling pathway. J Hematol Oncol. 2016;9:42. https://doi.org/10.1186/s13045-016-0269-y.

10. Wang T, et al. Comorbidities and multi-organ injuries in the treatment of COVID-19. Lancet. 2020;395:e52. https://doi.org/10.1016/50140-6736(20)30558-4.

\section{Publisher's Note}

Springer Nature remains neutral with regard to jurisdictional claims in published maps and institutional affiliations.

Ready to submit your research? Choose BMC and benefit from:

- fast, convenient online submission

- thorough peer review by experienced researchers in your field

- rapid publication on acceptance

- support for research data, including large and complex data types

- gold Open Access which fosters wider collaboration and increased citations

- maximum visibility for your research: over $100 \mathrm{M}$ website views per year

At BMC, research is always in progress.

Learn more biomedcentral.com/submissions 